http://dx.doi.org/10.18359/ravi.2017

\title{
Características de la investigación formativa en los programas de Ingeniería, a distancia y virtual, en Bogotá ${ }^{1}$
}

\author{
José Israel Huertas Zamudio² , Martín Elías Villamil Rozo $^{3}$ \\ Universidad Militar Nueva Granada, Colombia
}

Recibido, abril 24 de 2015

Concepto evaluación, junio 09 de 2016

Aceptado, junio 30 de 2016
Referencia: Huertas Zamudio, J.; Villamil Rozo, M. (2016). "Características de la investigación formativa en los programas de ingeniería, a distancia y virtual, en Bogotá". Revista Academia y Virtualidad, 9, (2), 121-137

\section{Resumen}

En este artículo se presentan los resultados del proyecto de investigación: "Caracterización de la investigación formativa de los programas de Ingeniería, ofrecidos mediante las metodologías a distancia (tradicional) y virtual, en la ciudad de Bogotá". El propósito consistió en describir los procesos de investigación formativa en los componentes: proyecto institucional (misión, visión, principios y horizonte institucional), estudiantes, docentes y procesos académicos. La información se recolectó mediante encuestas y entrevistas estructuradas y aplicadas de manera individual a directores participantes de 15 programas de Ingeniería. Entre los resultados se destacan: la relación explícita entre la investigación formativa y el PEI; la participación vivencial de los estudiantes en contextos reales y simulados; la participación y liderazgo de los profesores; la existencia de diseños curriculares de los programas que proponen estrategias, oportunidades y alternativas para favorecer la investigación formativa. En la discusión de resultados se reconoce la acción de instituciones como el Estado y los vínculos e interacciones entre el conocimiento y las acciones individuales (de los programas de Ingeniería y los estudiantes). Como conclusiones, los hallazgos aportan información sobre los procesos investigativos y las tendencias positivas de la investigación formativa de los programas de Ingeniería en Bogotá.

Palabras clave: : caracterización, investigación formativa, programas de ingeniería a distancia, programas virtuales.

\footnotetext{
${ }^{1}$ Artículo de investigación, resultado del proyecto "Caracterización de la investigación formativa de los programas de Ingeniería, ofrecidos mediante la metodología a distancia, en la ciudad de Bogotá”, adscrito a la Oficina de Planeación, en el proyecto Ciencia, tecnología e innovación académica, con el código 04.01.01, al programa de Ingeniería Industrial, Facultad de Estudios a Distancia, en la Universidad Militar Nueva Granada.

${ }^{2}$ Administrador de Empresas; Magíster Teoría Económica de la Universidad Nacional de Colombia; Especialista Dirección Estratégica y Prospectiva de Instituciones Universitarias; Profesor Asociado del programa de Ingeniería Industrial a Distancia, Universidad Militar Nueva Granada. jose.huertas@unimilitar. edu.co

${ }^{3}$ Ingeniero Industrial; Especialista Aprendizaje Autónomo; Magíster Pedagogía, Universidad Santo Tomas; Profesor Asociado, Programa Ingeniería Industrial a Distancia, Universidad Militar Nueva Granada. martin. villamil@unimilitar.edu.co
} 


\title{
Research training features: Distance \& virtual Engineering programs in Bogota
}

\begin{abstract}
This paper offers the results of the research project "Description of research training of Engineering programs through distance (or traditional) and virtual methodologies in Bogota." The main goal was to describe the research training processes of the relevant constituents: institutional project (mission, vision, principles and perspectives), students, teachers and academic processes. Data was collected through surveys and structured interviews conducted individually to school board directors of 15 Engineering programs. Among the results: the evident bond between formative research and IEP; experiential students' involvement in real and virtual contexts; the teachers' involvement and leadership; the implementation of curricular design of programs including strategies, opportunities and alternatives to encourage the research training. The discussion of results shows the involvement of official institutions and the links and interactions between individual knowledge and actions (Engineering programs and students.) Finally, findings provide information upon research processes and positive trends in research training of the Engineering programs in Bogotá.
\end{abstract}

Keywords: features, research training, distance engineering programs, virtual programs.

\section{Caraterísticas da pesquisa formativa nos programas de engenharia, a distância e virtual, em Bogotá}

\section{Resumo}

Neste artigo apresentam-se os resultados do projeto de pesquisa: "Caraterização da pesquisa formativa dos programas de Engenharia, oferecidos mediante as metodologias a distância (tradicional) e virtual, na cidade de Bogotá". O propósito consistia em descrever os processos de pesquisa formativa nos componentes: projeto institucional (missão, visão, princípios e horizonte institucional) estudantes, docentes, e processos académicos. A informação foi coletada por médio de enquetes e entrevistas estruturadas aplicadas individualmente a diretores participantes de 15 programas de Engenharia. Entre os resultados cabe destacar: a relação explicita entre pesquisa formativa e o PEI (projeto Educativo institucional, NDT); a participação vivencial dos estudantes nos contextos reais e simulados; a participação e liderança dos professores; a existência de desenhos curriculares dos programas que propõem estratégias, oportunidades e alternativas para favorecer a pesquisa formativa. $\mathrm{Na}$ discussão dos resultados se reconhece a ação das instituições como o Estado e os vínculos e interações entre conhecimento e as ações individuais (dos programas de engenharia e os estudantes). Enquanto as conclusões, os achados fornecem informação sobre os processos de pesquisa e as tendências positivas da pesquisa formativa dos programas de Engenharia em Bogotá.

Palavras chave: caraterização, pesquisa formativa, programas de engenharia a distância, programas virtuais. 


\section{Introducción}

Los resultados de este ejercicio aportan datos e información sobre la investigación formativa para la toma de decisiones que les permite a ingenieros y profesionales cumplir con las exigencias a fin de participar activamente en la sociedad del conocimiento, en el saber hacer y en la identificación y resolución de problemas (Castellanos, 2013), (Misas, 2004). Al respecto, es pertinente contar con los distintos aportes conceptuales de la investigación formativa.

Como antecedentes conceptuales se destacan el surgimiento de estrategias, planteamientos y conceptos clave sobre la investigación formativa. Por ejemplo, la investigación didáctica, mediante la cual los profesores pueden mejorar los procesos de aprendizaje (Parra, 2004); los planteamientos sobre el aprendizaje de la investigación (Restrepo, 2003); la propuesta de distintas estrategias para desarrollar la investigación formativa, como el aprendizaje basado en problemas ABP (Restrepo, 2014); el énfasis en el caso específico de la investigación formativa hacia la apropiación de conocimiento por parte de los estudiantes (Hernández, 2003). También se destaca el estudio de métodos asociados pertinentes con la investigación formativa para la creación de comunidades de aprendizaje en línea para adultos, a saber: enfoques en el entorno del estudiante, en problemas de la vida real y fomento del autoaprendizaje (Yagodzinski, 2014).

Para la consolidación de estos procesos, las universidades y los programas de Ingeniería deben contribuir con su apoyo irrestricto a los procesos de investigación formativa, para lo cual como todas las organizaciones en general, requieren disponer de datos, información y conocimientos a fin de apoyar la toma de decisiones (Castellanos, 2007).

Sin embargo, en relación con la disponibilidad de información sobre las características de los procesos de la investigación formativa, en el caso de los programas de Ingeniería de la metodología a distancia y la modalidad virtual, resulta importante mencionar que luego de efectuar una exploración bibliográfica, no se encuentra información suficiente acerca del tema relativo a la investigación formativa.

Con este propósito, se plantea la siguiente pregunta: ¿Cómo se desarrollan los procesos de investigación formativa articuladas con el currículo? Y se precisa como objetivo, describir los procesos de investigación formativa en los siguientes componentes (proyecto institucional: misión, visión, principios y horizonte institucional; estudiantes; docentes; procesos académicos) en los diferentes programas de Ingeniería, modalidades distancia (tradicional) y virtual, en Bogotá.

\footnotetext{
${ }^{4}$ Los programas se clasifican según los criterios de búsqueda del Sistema Nacional de Información de la Educación Superior (SNIES).

${ }^{5}$ En el Decreto 1295 de 2010, en el artículo 16, un programa a distancia "se caracteriza por utilizar estrategias de enseñanza-aprendizaje que permiten superar las limitaciones de espacio y tiempo entre los actores del proceso educativo". En el artículo 17, del mencionado Decreto, los programas virtuales, "adicionalmente, exigen el uso de las redes telemáticas como entorno principal, en el cual se lleven a cabo todas o al menos el ochenta por ciento $(80 \%)$ de las actividades académicas".
} 
Para desarrollar la investigación, a nivel metodológico se dispusieron de los siguientes elementos: diseño investigativo no experimental (observacional), dado que las variables que intervienen no pueden ser manejadas por los investigadores; el tipo de investigación es descriptiva correlacional, las variables son descriptivas sin atribuir causalidad; la investigación se considera ex-post-facto, es decir, que los datos e información provienen de hechos sucedidos.

Como participantes se indican 15 directores de sendos programas de Ingeniería, de un total de 18 programas en la ciudad de Bogotá, tal como se muestra en la tabla 1. Cada una de las personas diligenció una encuesta y participó en una entrevista estructurada individual.

Tabla 1. Programas de Ingenieria. Participantes ${ }^{4}$

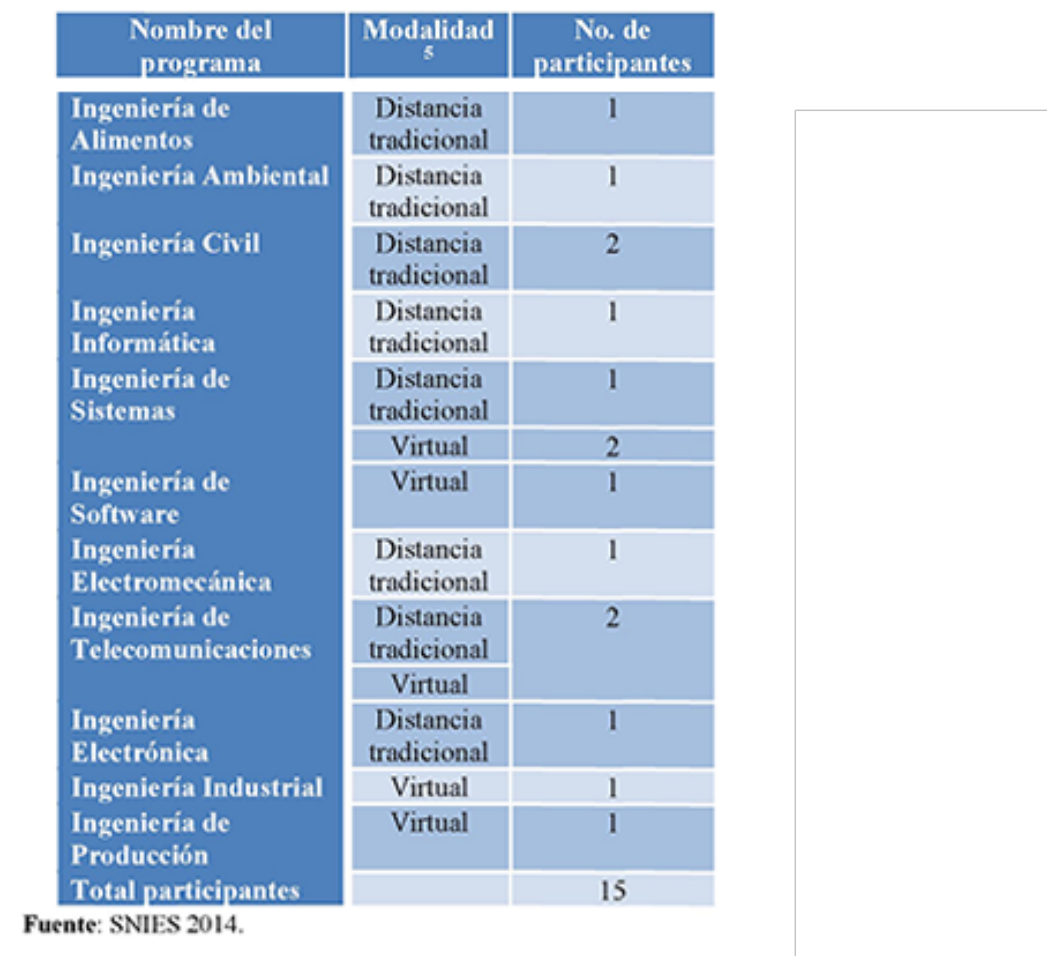

El artículo presenta además de la introducción, tres secciones: en la primera se exponen los resultados; en segundo término, se discuten los resultados; en la tercera sesión se indican las conclusiones.

\section{Resultados}

En seguida se presentan los resultados relevantes relacionados con los procesos de investigación formativa, expresada en los componentes: proyecto institucional, estudiantes, docentes y procesos académicos. Se incluyen datos sobre los semilleros, las líneas y grupos de investigación, y los paquetes de software especializado.

\section{Componente del proyecto institucional: misión, visión, principios}

Este referente indaga sobre la filosofía y los principios del programa; la visión y la misión del programa; el PEI de la Universidad. Tal como se muestra en la figura 1, se contempla la 
investigación formativa en la visión del programa en un 93\%. De otra parte, en la tabla 2 se halla que entre los programas consultados, 11 de ellos, equivalente al $73 \%$, están de acuerdo en que el PEI de la Universidad distingue de manera explícita la investigación formativa. Respecto a la filosofía y los principios del programa aluden explícitamente a la investigación formativa como elemento básico del proceso académico, en un 53\% (8 programas); llama la atención la percepción parcialmente de acuerdo equivalente al 40\% (6 programas).

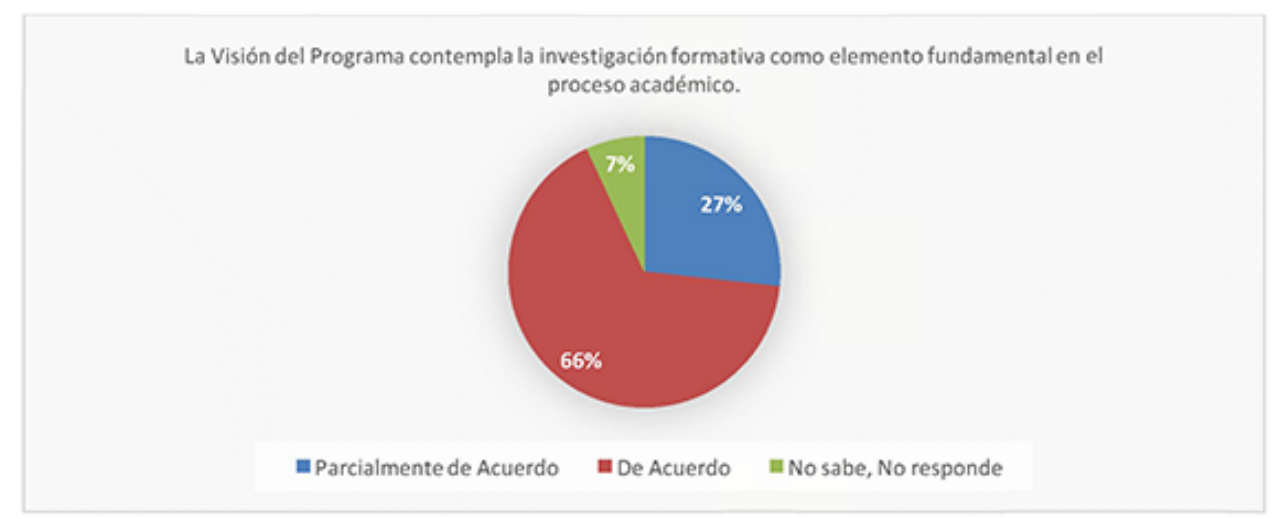

Figura 1. Visión de los programas.

Tabla 2. Componente: misión, visión, principios.



Fuente: Elaboración propia con base en los datos disponibles del proyecto de investigación.

\section{Componente de estudiantes}

En los programas, 93\% (14) perciben que las asignaturas contribuyen al proceso de aprendizaje con reflexiones sobre el contexto, tal como lo muestra la figura 2.

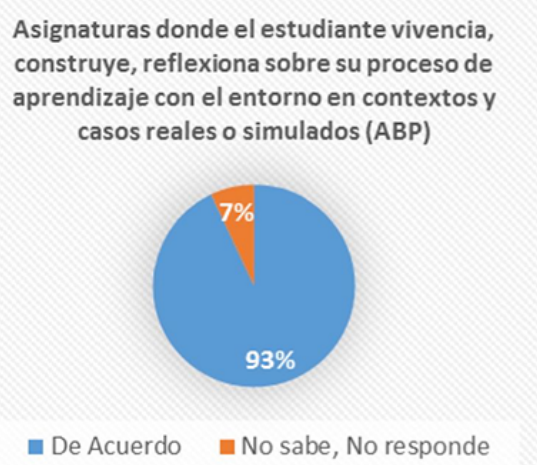

Figura 2. Asignaturas 
Este referente explora sobre la experiencia de la investigación formativa; el conocimiento de las herramientas de investigación formativa; los valores agregados en el proceso de formación profesional; la experiencia que ha contribuido al desarrollo de competencias, habilidades y destrezas, los conocimientos de experiencias.

En la tabla 3 se observa que el 80\% (12 programas) conocen experiencias de ejercicios de investigación formativa aplicadas en el programa académico. Sin embargo, al evaluar la experiencia de investigación formativa como parte importante o considerable en la ponderación de la calificación de alguna de las asignaturas que propone el plan de estudios del programa, el $53 \%$ ( 8 programas) están de acuerdo, mientras que el $40 \%$ (6 programas) manifiestan su desacuerdo parcial.

Tabla 3. Componente: estudiantes

\begin{tabular}{|c|c|c|c|c|}
\hline fitems & $\begin{array}{c}\text { En } \\
\text { desacuerdo }\end{array}$ & $\begin{array}{l}\text { Parcialmente } \\
\text { de acuerdo }\end{array}$ & $\begin{array}{c}\text { De } \\
\text { acuerdo }\end{array}$ & $\begin{array}{l}\text { No sabe, no } \\
\text { responde }\end{array}$ \\
\hline $\begin{array}{l}\text { Conoce experiencias relacionadas con el ejercicio } \\
\text { de la investigación formativa aplicadas en el } \\
\text { programa académico }\end{array}$ & & 2 & 12 & 1 \\
\hline $\begin{array}{l}\text { Se evalúa la experiencia de investigación } \\
\text { formativa como parte importante o considerable } \\
\text { en la ponderación de la califícación de alguna de } \\
\text { las asignaturas que propone el plan de estudios } \\
\text { del programa }\end{array}$ & & 6 & 8 & 1 \\
\hline $\begin{array}{l}\text { La investigación formativa ha propiciado valores } \\
\text { agregados en el proceso de formación profesional }\end{array}$ & & 3 & 11 & 1 \\
\hline $\begin{array}{l}\text { Conoce las herramientas de la investigación } \\
\text { formativa y las aplica en el desarrollo de las } \\
\text { asignaturas que constituyen el plan de estudios del } \\
\text { programa al que pertenece }\end{array}$ & & 4 & 10 & 1 \\
\hline
\end{tabular}

\section{Componente de los docentes}

Este referente examina si los docentes estudian pedagogías específicas; si utilizan el constructivismo, las corrientes pedagógicas conductistas o el aprendizaje significativo; si conocen los elementos epistemológicos y metodológicos que tipifican la investigación formativa.

Los docentes participantes, en $60 \%$ están de acuerdo y consideran la investigación formativa como elemento básico en la formación profesional, mientras que 33\% (5) manifiesta su desacuerdo parcial; esto se muestra en la figura 3. Igualmente, se evidencian liderazgos de los docentes, al frente de grupos y/o semilleros de investigación, en $66 \%$, quienes aplican o desarrollan experiencias de investigación formativa; entre tanto, $27 \%$ (4) expresa su desacuerdo parcial, tal como se indica en la figura 4. Los docentes manifiestan en $73 \%$ que la investigación formativa propicia valores agregados en el proceso de formación profesional del estudiante; sin embargo, 20\% (3) manifiesta su desacuerdo parcial, tal como se aprecia en la tabla 4. En el ítem sobre aplicación frecuente de herramientas en los procesos de enseñanzaaprendizaje en las asignaturas, 53\% (8) están de acuerdo; es interesante observar que $40 \%$ (6) indica su desacuerdo parcial, tal como se presenta en la tabla 4.

Sobre la capacitación relacionada con la construcción, aplicación y desarrollo de las herramientas de investigación formativa, $40 \%$ (6) están de acuerdo; no obstante, 53\% (8) manifiesta su desacuerdo parcial, tal como aparece en la tabla 4. En relación con el uso de pedagogías conductistas, $40 \%$ (6) está 
en desacuerdo; por el contrario, se evidencian los acuerdos con elementos conceptuales del constructivismo en $73 \%$ (11); han tomado parte en el direccionamiento, monitoria, seguimiento y evaluación de acuerdo en un 87\% (13); han participado en la elaboración de proyectos investigativos en $67 \%$ (10), tal como se enuncia en la tabla 4.

Los docentes del programa consideran la investigación formativa como un elemento básico y fundamental en el proceso de formación profesional.

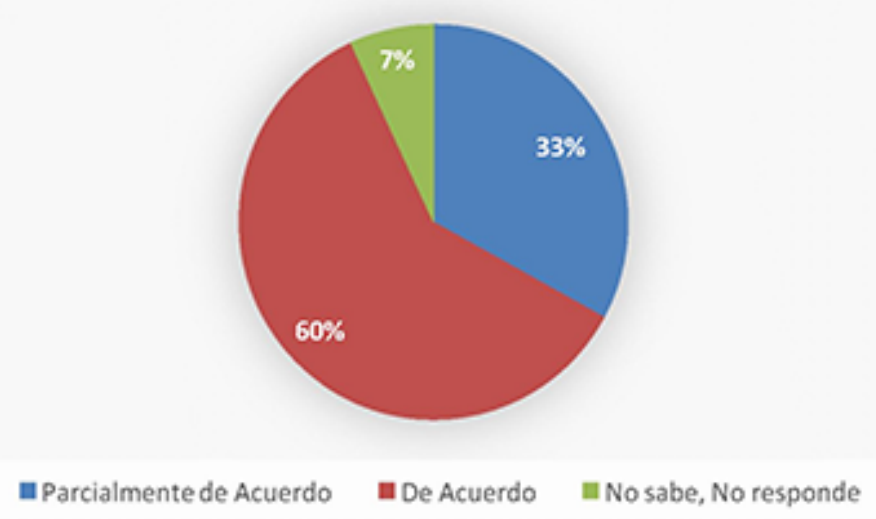

Figura 3. Docentes que consideran la investigación formativa como un elemento básico. Fuente: Elaboración propia con base en los datos disponibles del proyecto de investigación.



Figura 4. Lideres en grupos y/o semilleros de investigación.

Fuente: Elaboración propia con base en los datos disponibles del proyecto de investigación. 
Tabla 4. Componente: docentes e investigación formativa

\begin{tabular}{|c|c|c|c|c|}
\hline Ifem & $\underline{\text { En }} \underline{\underline{\text { desacuerdo }}}$ & $\frac{\text { Parcialmente }}{\underline{\text { de acuerdo }}}$ & $\underline{\text { De }}$ & $\frac{\text { No sabe, no }}{\underline{\text { responde }}}$ \\
\hline $\begin{array}{l}\text { La investigación formativa ha propiciado valores } \\
\text { agregados en el proceso de formación profesional } \\
\text { de sus estudiantes }\end{array}$ & & 3 & 11 & 1 \\
\hline $\begin{array}{l}\text { Se aplican con frecuencia las herramientas de la } \\
\text { investigación formativa en los procesos de } \\
\text { enseñanza-aprendizaje en las asignaturas que } \\
\text { imparten }\end{array}$ & & 6 & 8 & 1 \\
\hline $\begin{array}{l}\text { Se recibe capacitación relacionada con la } \\
\text { construcción, aplicación y desarrollo de las } \\
\text { herramientas de la investigación formativa, por } \\
\text { parte del programa al que pertenece }\end{array}$ & & 8 & 6 & 1 \\
\hline $\begin{array}{l}\text { Se lideran en la actualidad procesos o actividades } \\
\text { relacionados con el cjercicio de la investigación } \\
\text { formativa }\end{array}$ & 1 & 5 & 8 & 1 \\
\hline $\begin{array}{l}\text { Se han elaborado proyectos (por lo menos uno) de } \\
\text { investigación formativa a lo largo del desarrollo } \\
\text { de su vida académica }\end{array}$ & I & 1 & 12 & 1 \\
\hline $\begin{array}{l}\text { Ha participado en la elaboración de proyectos } \\
\text { investigativos que contemplen la investigación } \\
\text { formativa como elemento básico en su diseño }\end{array}$ & 1 & 3 & 10 & 1 \\
\hline $\begin{array}{l}\text { Ha participado en el direccionamiento, monitoria, } \\
\text { seguimiento, evaluación o calificación de } \\
\text { situaciones de investigación formativa ( } \mathrm{v} \text {.g., } \\
\text { trabajos de grado como monografias, proyectos } \\
\text { de investigación, tesis, etc.) }\end{array}$ & & 1 & 13 & 1 \\
\hline $\begin{array}{l}\text { Se conocen los elementos epistemologicos y } \\
\text { metodologicos que tipifican la investigación } \\
\text { formativa, y la diferencian de las otras formas, } \\
\text { clases y niveles de investigación }\end{array}$ & 1 & 7 & 6 & 1 \\
\hline $\begin{array}{l}\text { Se utilizan el aprendizaje significativo en la } \\
\text { impartición de sus asignaturas }\end{array}$ & & 2 & 12 & 1 \\
\hline $\begin{array}{l}\text { Se utilizan corrientes pedagogicas conductistas } \\
\text { (docente como eje central en el proceso de } \\
\text { enseñanza-aprendizaje) en la impartición de sus } \\
\text { asignaturas. }\end{array}$ & 6 & 5 & 3 & 1 \\
\hline $\begin{array}{l}\text { Se utilizan el construetivismo (estudiante como } \\
\text { eje central en el proceso de enseñanza- } \\
\text { aprendizaje) como corriente pedagógica en la } \\
\text { impartición de sus asignaturas. }\end{array}$ & & 3 & 11 & 1 \\
\hline
\end{tabular}

Futente: Elaboración propia con base en los datos disponibles del proyecto de investigación.

\section{Componente de procesos académicos}

Este referente explora los elementos relacionados con los procesos académicos. Al respecto, no se contempla la investigación formativa como electiva (seminario) en el plan de estudios con un desacuerdo de 60\% (9); la investigación formativa cumple un papel transversal en el diseño curricular de acuerdo equivalente a 53\% (8), mientras que 40\% (6) manifiesta desacuerdo parcial; existen semilleros, grupos de interés en 67\% (10); la participación en encuentros, seminarios, coloquios a nivel internacional no es satisfactoria para $40 \%$ (6); existe acuerdo con la articulación de las investigaciones con el sector productivo y la sociedad en $47 \%$ (7), mientras que 47\% (7) se muestran parcialmente de acuerdo; existen desacuerdos sobre guías, 
folletos, ayudas didácticas en 13\%; la experiencia de la investigación formativa se contempla como elemento básico y obligatorio de acuerdo para 53\%, mientras que $33 \%$ indica su acuerdo parcial.

En los programas consultados, tal como se muestra en la tabla 5, 67\% (10) consideran su acuerdo que el diseño curricular del programa propone estrategias, actividades y recursos, y otras alternativas y oportunidades para el ejercicio de la investigación formativa.

Tabla 5. Componente: procesos académicos
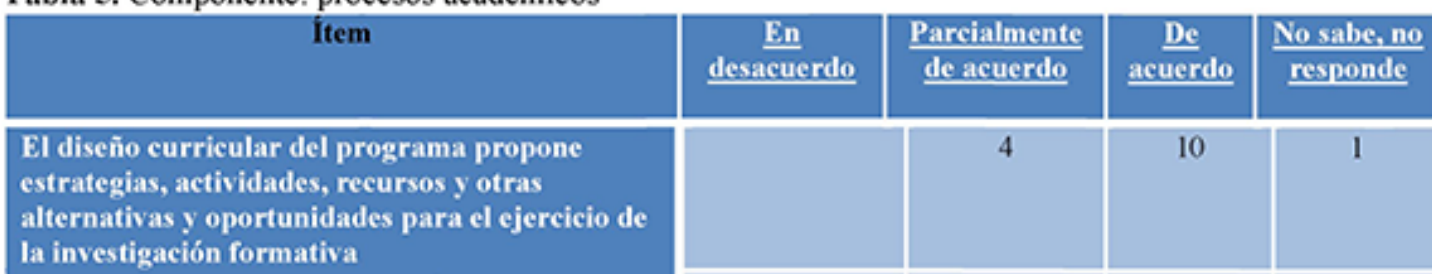

la investigación formativa

La experiencia de la investigación formativa se

contempla como elemento básico y obligatorio en

el ejercicio académico del programa

Existen guías, folletos, ayudas didácticas, entre

otros materiales, que contribuyan al ejereicio de

la investigación formativa al interior del

programa

Existe articulación de las investigaciones en

marcha con el sector productivo y la sociedad en

busca de lograr niveles óptimos de desarrollo por

medio de la promoción de actividades de

aplicación y apropiación del conocimiento

Ha participado en encuentros, seminarios,

coloquios, foros, congresos, a nivel nacional, en

los que el tema central ha sido la investigación

formativa

Ha participado en encuentros, seminarios,

coloquios, foros, congresos, a nivel internacional,

en los que el tema central ha sido la investigación

formativa

Existen semilleros, grupos de interés o grupos

interdisciplinares que propicien, faciliten y/o

desarrollen ejercicios de investigación formativa

La investigación formativa cumple un papel

transversal en el diseño curricular del programa

Se contempla la investigación formativa como

una electiva (seminario) al interior del plan de

estudios del programa

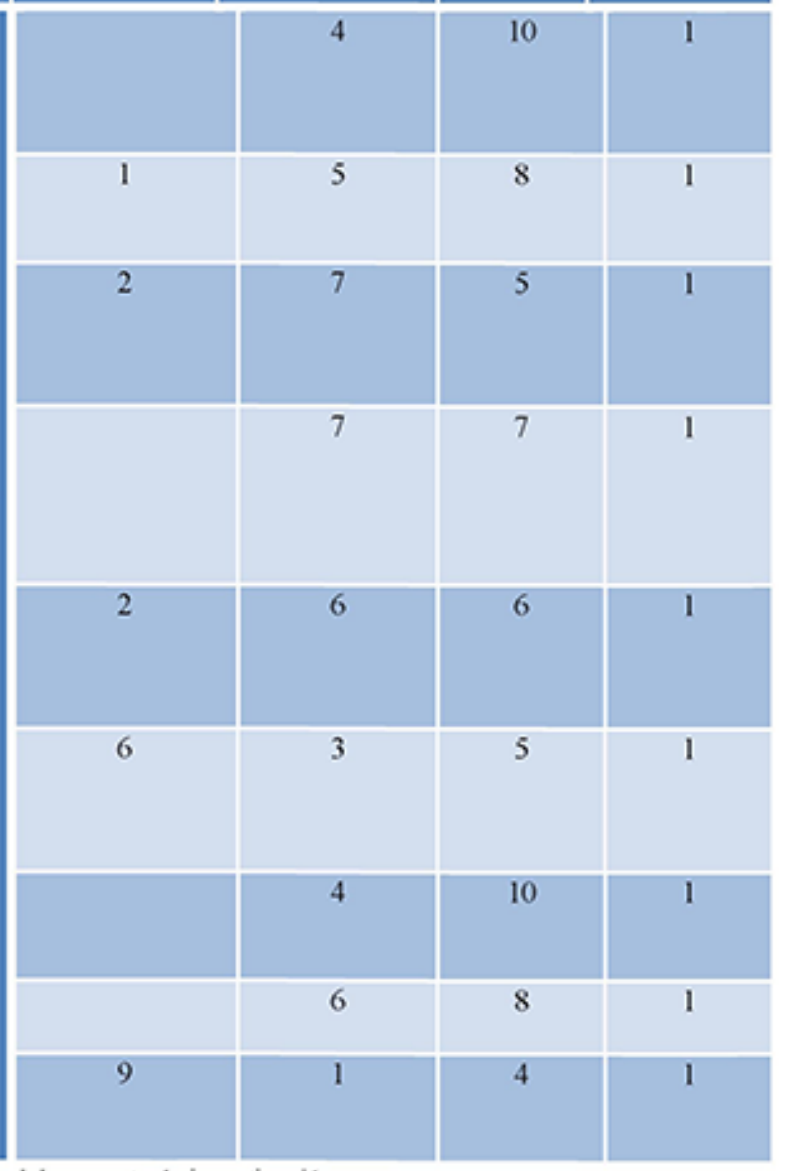

Fuente: Elaboración propia con base en los datos disponibles del proyecto de investigación.

\section{Resultados complementarios sobre los procesos académicos}

En el componente de procesos académicos se destacan los semilleros de investigación, las líneas de investigación, los grupos de investigación y los paquetes de software especializado. A continuación se enuncian los resultados relacionados con estos elementos. En la tabla 6, los programas participantes reportan 27 semilleros de investigación. 
Tabla 6. Semilleros de investigación

\begin{tabular}{|c|c|}
\hline Nombre del programa & Nombre del semillero \\
\hline \multirow{4}{*}{ Ingeniería de Alimentos } & VITAL \\
\hline & Biotecol \\
\hline & Creci 3 \\
\hline & Gestión Ambiental \\
\hline \multirow{4}{*}{ Ingeniería Ambiental } & Biodiversidad (manejo de Suelos) \\
\hline & Sostenibilidad ambiental \\
\hline & Energías alternativas \\
\hline & Semillero 1 \\
\hline \multirow[t]{2}{*}{ Ingeniería Civil } & Trabajos generales de ingenieros \\
\hline & Atención y prevención del desastre \\
\hline \multirow[t]{3}{*}{ Ingeniería Informática } & Greentic \\
\hline & Innovapp \\
\hline & Virtualización 3D \\
\hline \multirow[t]{3}{*}{ Ingeniería de Sistemas } & Robótica y telecomunicaciones \\
\hline & Procesos de software libre \\
\hline & Informática educativa \\
\hline \multirow[t]{2}{*}{ Ingeniería de Software } & Movilidad \\
\hline & Contra movilidad \\
\hline \multirow{3}{*}{$\begin{array}{l}\text { Ingeniería } \\
\text { Electromecánica }\end{array}$} & Computación Móvil \\
\hline & Procesamiento de imágenes \\
\hline & Videojuegos \\
\hline \multirow[t]{2}{*}{ Ingeniería Telecomunicaciones } & Ingeniería de software \\
\hline & Ingeniería de telecomunicaciones \\
\hline \multirow{2}{*}{ Ingeniería Industrial } & Semillero LIPEX \\
\hline & TGI \\
\hline \multirow[t]{2}{*}{ Ingeniería de Producción } & RSE \\
\hline & IMPROVE \\
\hline
\end{tabular}

Fuente: Elaboración propia. 
Aquí se reportan datos relacionados con las líneas de investigación y áreas de estudio: 60\% (9) de los programas participantes están de acuerdo con los grupos de investigación, tal como se muestra en la figura 6. Las líneas de investigación están adscritas a áreas de estudio, excepto en los programas de Ingeniería de Alimentos, los cuales se enfocan en las áreas de estudio, tal como se indica en la tabla 7.



Figura 5. Grupos de investigación.

Fuente: Elaboración propia. 
Tabla 7. Lineas de investigación

\begin{tabular}{|c|c|c|}
\hline Nombre del programa & Líneas de investigación & Areas de estudio \\
\hline \multirow{4}{*}{ Ingenieria Alimentos } & & Desarrollo Agroindustrial \\
\hline & & Procesos biotecnológicos \\
\hline & & $\begin{array}{l}\text { Evaluación y calidad de materias } \\
\text { primas y productos }\end{array}$ \\
\hline & & $\begin{array}{l}\text { Seguridad y gestión de la calidad } \\
\text { alimentaria }\end{array}$ \\
\hline \multirow[t]{7}{*}{ Ingenieria Ambiental } & Manejo de residuos & Quimica \\
\hline & Ecoeficiencia & Quimica \\
\hline & Manejo y tratamiento de aguas & Biologia y Química \\
\hline & Energias renovables & Fisica y Química \\
\hline & $\begin{array}{l}\text { Control de la contaminación } \\
\text { atmosférica }\end{array}$ & Fisica y Matemáticas \\
\hline & Pavimentos & Pavimentación \\
\hline & Movilidad & \\
\hline \multirow[t]{3}{*}{ Ingenieria Civil } & Contramovilidad & \\
\hline & Trabajos generales & \\
\hline & Atención y prevención de desastres & \\
\hline \multirow[t]{8}{*}{ Ingenieria Informática } & Desarrollo de software & Ingenieria \\
\hline & Telemática & Ingenieria \\
\hline & Ciencias computación & Interfaz hombre/máquina \\
\hline & Ingenieria software & Desarrollo vertical \\
\hline & Telemática, telecomunicaciones & Redes Corporativas \\
\hline & Emprendimiento & \\
\hline & Gerencia & \\
\hline & Tecnologia de información & \\
\hline \multirow{3}{*}{ Ingenieria de Sistemas } & Gestión y diseño de procesos & \\
\hline & Gestión de sistemas & \\
\hline & Ingenieria de software & \\
\hline \multirow{7}{*}{$\begin{array}{l}\text { Ingenieria de Software } \\
\text { Ingenieria Electromecánica }\end{array}$} & Ingenicria de software & Software \\
\hline & Big Data & \\
\hline & Computación móvil & \\
\hline & Construcción de videojucgos & \\
\hline & Inteligencia artificial & \\
\hline & $\begin{array}{l}\text { Mctodos computacionales para } \\
\text { bioingenieria }\end{array}$ & \\
\hline & $\begin{array}{l}\text { Aplicaciones sobre redes de banda } \\
\text { ancha }\end{array}$ & Cloud computing, como servicio \\
\hline \multirow[t]{5}{*}{$\begin{array}{l}\text { Ingenieria de } \\
\text { Telecomunicaciones }\end{array}$} & $\begin{array}{l}\text { Seguridad en redes de } \\
\text { telecomunicaciones }\end{array}$ & Auditoria, politicas y niveles \\
\hline & Integración de servicios móviles & Desarrollo de contenidos \\
\hline & $\begin{array}{l}\text { Automatización y herramientas } \\
\text { logicas }\end{array}$ & \\
\hline & $\begin{array}{l}\text { Desarrollo de infraestructura } \\
\text { tecnologica }\end{array}$ & \\
\hline & Desarrollo de Software & \\
\hline \multirow[t]{5}{*}{ Ingenieria Electrónica } & Infracstruetura y redes & \\
\hline & Dispositivos y herramientas logicas & \\
\hline & Competitividad industrial & $\begin{array}{l}\text { Finangas, Planeación y Control de } \\
\text { producción, Disctio industrial, } \\
\text { Logística de mereados. }\end{array}$ \\
\hline & Productividad industrial & $\begin{array}{l}\text { Ingenicria de metodos, diseno de } \\
\text { sistemas productivos, gestión del } \\
\text { talento humano. }\end{array}$ \\
\hline & Innovación y educación industrial & $\begin{array}{l}\text { Diseno industrial, logistica de } \\
\text { mereados, gestión tecnologica, } \\
\text { planeación y control de producción, } \\
\text { Ingenicria logistica. }\end{array}$ \\
\hline Ingenieria de Producción & Desarrollo tecnologico & Ingenieria \\
\hline
\end{tabular}

Fucnte: Elaboración propia. 
Asimismo, cada programa reporta información sobre grupos de investigación adscritos a una área disciplinar, tal como se observa en la tabla 8.

Tabla 8. Grupos de investigación

\begin{tabular}{|c|c|c|}
\hline Nombre del programa & Grupo de investigación & Área \\
\hline \multirow[t]{3}{*}{ Ingeniería de Alimentos } & Creci & \multirow{2}{*}{$\begin{array}{l}\text { Macrolínea de producción de } \\
\text { alimentos }\end{array}$} \\
\hline & Biotics & \\
\hline & $\begin{array}{l}\text { Agroforestería y } \\
\text { Biodiversidad tropical }\end{array}$ & Desarrollo sostenible \\
\hline \multirow[t]{4}{*}{ Ingeniería Ambiental } & Biotecnologia & Agroindustria Rural \\
\hline & Inyumacizo & Innovación Ambiental \\
\hline & $\begin{array}{l}\text { Grupo de Estudios } \\
\text { Regionales Quinini }\end{array}$ & Educación Ambiental \\
\hline & 1 Grupo reconocido & Pavimento \\
\hline Ingeniería Civil & Ingeniería civil a distancia & 4 áreas \\
\hline \multirow[t]{4}{*}{ Ingeniería Informática } & $\begin{array}{l}\text { Gestión en ciencia, } \\
\text { innovación, tecnologia y } \\
\text { organizaciones }\end{array}$ & Ciencia y tecnología \\
\hline & G115 & Educación \\
\hline & Entrepreneurship Group & Emprendimiento \\
\hline & G3 Pymes & Gerencia \\
\hline \multirow{5}{*}{ Ingeniería Sistemas } & Ontare & Ingeniería \\
\hline & Byte in design & $\begin{array}{l}\text { Ing. de software y } \\
\text { simulación }\end{array}$ \\
\hline & Guane & $\begin{array}{l}\text { Ing. de software-mundos } \\
\text { virtuales }\end{array}$ \\
\hline & Davinci & \\
\hline & Innovación tecnológica & \\
\hline \multirow[t]{2}{*}{ Ingeniería Software } & Gitis & $\begin{array}{l}\text { Software y } \\
\text { telecomunicaciones }\end{array}$ \\
\hline & Informática educativa & Educación \\
\hline \multirow[t]{2}{*}{ Ingeniería Electromecánica } & SYSCOMP & $\mathrm{Ns} / \mathrm{Nr}$ \\
\hline & G 115 & Ingenieria \\
\hline \multirow[t]{2}{*}{$\begin{array}{l}\text { Ingeniería } \\
\text { Telecomunicaciones }\end{array}$} & Davinci & $\begin{array}{l}\text { Desarrollo de infraestructura } \\
\text { y software }\end{array}$ \\
\hline & Cedester & Automatización \\
\hline \multirow[t]{3}{*}{ Ingeniería Electrónica } & $\mathrm{Ns} / \mathrm{Nr}$ & Control simuladores \\
\hline & & Agronica \\
\hline & & Robótica \\
\hline Ingeniería Industrial & INAMPE & $\begin{array}{l}\text { Productividad, } \\
\text { competitividad }\end{array}$ \\
\hline
\end{tabular}

Fuente: Elaboración propia.

Para apoyar los diferentes procesos de investigación formativa se dispone de software especializado. En la figura 6, los programas se muestran de acuerdo a esta realidad en $60 \%$. En la tabla 9 se muestran los detalles relacionados con el software que disponen los programas. 


\section{Bases de datos especializadas y reconocidas en el programa}

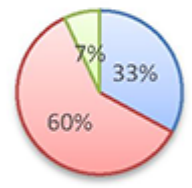

$\square$ Parcialmente de acuerdo $\square$ De acuerdo $\square$ No sabe, no responde

Figura 6. Bases de datos especializadas.

Fuente: Elaboración propia.

Tabla 9. Paquetes de software especializado que maneja el programa

\begin{tabular}{|c|c|c|}
\hline Nombre del programa & Paquete & A reas de cubrimiento \\
\hline \multirow[t]{3}{*}{ Ingeniería Ambiental } & Autocad & $\begin{array}{l}\text { Mapificación, } \\
\text { Fotointerpretación }\end{array}$ \\
\hline & Arcgis & Diseño de plantas y Equipos \\
\hline & Vissim & Tránsito y transporte \\
\hline \multirow{3}{*}{ Ingeniería Civil } & Autocad & Modelamiento \\
\hline & Arijés & Topografia \\
\hline & Project & Administración \\
\hline \multirow[t]{15}{*}{ Ingeniería Informática } & Java (Netbases) & Desarrollo \\
\hline & Dw & Lógica \\
\hline & Post. Gres & Bases de datos \\
\hline & Packecttracer & Telemática \\
\hline & Logisim & Telemática \\
\hline & SQL & Bases de datos \\
\hline & PHP & Desarrollo de software \\
\hline & Tecnologia ORACLE & Base de datos \\
\hline & Microsoft & Desarrollo de software \\
\hline & Oracle & Bases de datos \\
\hline & Cisco & Redes \\
\hline & Evolutium & Dinámica de sistemas \\
\hline & LabVIEW & Arquitectura de computadores \\
\hline & Packettracer & Sistemas distribuidos \\
\hline & $\mathrm{C}++$ & Lógica \\
\hline \multirow{4}{*}{ Ingeniería de Sistemas } & MSX- 88 & Arquitectura de computadores \\
\hline & My SQL & Bases de datos \\
\hline & Software libre & $\mathrm{Ns} / \mathrm{Nr}$ \\
\hline & Pentaho & Mineria de datos (Weka) \\
\hline \multirow[t]{2}{*}{ Ingenieria de Software } & Java, Javascript & Disciplinares \\
\hline & Eclipse, Psint, Msql & \\
\hline \multirow{7}{*}{$\begin{array}{l}\text { Ingeniería } \\
\text { Electromecánica }\end{array}$} & Mathlab & Simulación \\
\hline & $\begin{array}{l}\text { Insight Segmentation and } \\
\text { Registration }\end{array}$ & Procesamiento de imágenes \\
\hline & $\begin{array}{l}\text { UCL Camino diffusion HRI } \\
\text { Toolbit }\end{array}$ & $\mathrm{Ns} / \mathrm{Nr}$ \\
\hline & $\begin{array}{l}\text { SPM Software- statistical } \\
\text { parametric Mapping }\end{array}$ & $\mathrm{Ns} / \mathrm{Nr}$ \\
\hline & MRI Cron & $\mathrm{Ns} / \mathrm{Nr}$ \\
\hline & LabVIEW & Electrónica \\
\hline & Multisim & Electrónica \\
\hline \multirow{8}{*}{$\begin{array}{l}\text { Ingenieria de } \\
\text { Telecomunicaciones }\end{array}$} & Packettracer & Redes \\
\hline & GNS 3 & Redes \\
\hline & Convenio dreamspark & Sistemas \\
\hline & Packettrasser & Redes y Telecomunicaciones \\
\hline & MathLab & $\begin{array}{l}\text { Telecomunicaciones y } \\
\text { Telemetria }\end{array}$ \\
\hline & LabVIEW & $\begin{array}{l}\text { Telecomunicaciones y } \\
\text { Telemetria }\end{array}$ \\
\hline & LabVIEW & Electrónica industrial \\
\hline & Mathlab & Ciencias básicas \\
\hline \multirow[t]{3}{*}{ Ingeniería Electrónica } & Proteus & Diseño y simulación de circuitos \\
\hline & Altium & Diseño de PCB \\
\hline & Robocell & Robótica y CMC \\
\hline Ingenieria Industrial & $\begin{array}{l}\text { Win QSB, } \\
\text { GAMS, } \\
\text { Visual manufacturing, } \\
\text { Promodel }\end{array}$ & $\begin{array}{l}\text { Investigación operativa I y } \\
\text { II, Procesos de fabricación } \\
\text { logistica, Ingenieria de } \\
\text { Producción. }\end{array}$ \\
\hline $\begin{array}{l}\text { Ingeniería de } \\
\text { Producción }\end{array}$ & $\begin{array}{l}\text { Para cada una de los bloques } \\
\text { temáticos }\end{array}$ & $\begin{array}{l}\text { Desde primer semestre. } \\
\text { Cubrimiento total }\end{array}$ \\
\hline
\end{tabular}




\section{Discusión de resultados}

En los resultados de los procesos de investigación formativa de los programas de Ingeniería se reconoce la acción de instituciones como el Estado para impulsar dichos procesos. Este planteamiento coincide con lo expresado por Morales (2014), "Las instituciones ayudan a formar expectativas colectivas y a coordinar actividades entre agentes con capacidades cognitivas distintas". Esta ayuda para superar obstáculos de distinta índole no es reciente; por ejemplo, las dificultades para la formación de los ingenieros colombianos desde el siglo XIX. En esa época, las condiciones no eran favorables (Safford, 1989): “[...] la economía pobre y atrasada de Colombia ofrecía pocas oportunidades para la actividad económica y demandaba aún menos los servicios de individuos técnicamente capacitados" (p. 31).

Asimismo, el Estado aparece como una de las instituciones principales que, como sugiere (Kalmanovitz, 2001), "puede comprometerse hacia la educación universal y a financiar parte de la investigación básica y aplicada de las universidades". No obstante, North, citado por (Kalmanovitz, 2001), advierte que no se debe tomar este rol del Estado como dado; en vez de ello es conveniente considerarlo como parte del proceso de desarrollo (p. 53). En este orden de ideas, de acuerdo con (García-Cabrera A. , 2015) se distinguen tres dimensiones para las instituciones, cada una de las cuales aporta al desarrollo de los procesos de investigacion formativa, así:

-ElEstado, en cuanto a la dimensión regulativa: en la Constitución Nacional, en el artículo 27, garantiza las libertades de enseñanza, aprendizaje, investigación y cátedra; en la Ley 30/1991, en el artículo 9, los programas de pregrado preparan para el desempeño de ocupaciones; en el decreto 1295/2010), en el artículo 5.5.1. "El programa debe prever la manera cómo va a promover la formación investigativa de los estudiantes o los procesos de investigación..."

- La dimensión normativa que influye en la investigacion formativa, evidente en el modelo de autoevaluación, propuesto por el Consejo Nacional de Acreditación, CNA.

- La dimensión cognitiva, expresada en las universidades y en sus programas de Ingeniería a través de los semilleros de investigación.

Asimismo, se evidencian los vínculos entre el conocimiento y las interacciones individuales. Estas relaciones son pertinentes por cuanto las Universidades y sus programas de Ingeniería son actores principales en la sociedad del conocimiento y los estudiantes. Para (García-Cabrera, 2015) y otros: "[...] son las instituciones las que rigen las interacciones humanas y condicionan el comportamiento tanto de las personas como de las organizaciones" (pp. 133-156).

Esta posibilidad entonces facilita la exploración de desarrollos significativos de investigación formativa con la influencia de las instituciones cognitivas sobre el individuo (estudiante) y los insumos de información requeridos en su proceso de formación. Por ejemplo, a partir de las ideas expuestas por Morales, se pueden incluir como insumos de información, los siguientes elementos:

- Las unidades administrativas, como la vicerrectorías de investigación, a nivel institucional.

- Los centros de investigación en las facultades y en los programas de Ingeniería.

- Las interacciones pasadas y actuales, por ejemplo, la vinculación laboral de los estudiantes facilitada por la metodología a distancia y virtual, que lleven a los profesionales a la toma de decisiones de cooperación para solucionar problemas de impacto colectivo en sus organizaciones.

- Los desarrollos de investigación (formativa) que aporten elementos para la solución de problemas, desde la pedagogía activa (los métodos ABP y AOP, los estudios de caso) y que contribuyan a disponer de escenarios de aprendizaje profundo, en vez de los escenarios superficiales $\mathrm{y} / \mathrm{o}$ estratégicos (donde el objetivo consiste prioritariamente en lograr una calificación), en los términos planteados 
por el profesor (Bain, 2007) y (Bain, 2014).

Así, en el conjunto de los resultados encontrados, se reconocen dos aspectos: la intervención estatal y el acatamiento de las condiciones básicas para el otorgamiento o renovación de los registros calificados. En tal sentido, en los programas participantes se evidencia el cumplimiento de los requisitos planteados en el Decreto 1295 de 2010. Por consiguiente, resulta importante fortalecer y controlar, en los diseños curriculares, los elementos de investigación formativa con el propósito de evitar "fugas" de conocimiento, tal como lo sugiere (Perkins, 2013). La existencia y dinámica de los semilleros, reconocida en $67 \%$, sigue los planteamientos realizados desde las teorías de representaciones sociales (Padilla Beltrán, Rincón Caballero, \& Buitrago Duarte, 2015), en cuanto a las posibilidades de contribuir a la construcción de pares profesionales "en el conocimiento práctico del ejercicio de la investigación".

\section{Conclusiones}

En este ejercicio investigativo se identificaron las características principales de los procesos de la investigación formativa de los programas de Ingeniería participantes, en Bogotá. De una parte, la injerencia del Estado, a través de la normatividad vigente, contribuye y aporta al fortalecimiento de la investigación formativa mediantela acción conjuntade las instituciones, en los programas de Ingeniería involucrados en el estudio, en el logro de efectos positivos en la contribución a la solución cooperada de problemas, en particular como coordinadores de las instituciones y en las personas, en los procesos de registros calificados y acreditación institucional. Los resultados disponibles sobre esta modalidad de programas aportan insumos para la realización de estudios comparativos con otros programas de Ingeniería, por ejemplo, los de metodología presencial.

De otra parte, los resultados contribuyen con evidencias sobre los sentidos y significados didácticos que tiene la investigación formativa en el ámbito pedagógico del aprendizaje para eventuales decisiones en cuanto a la solución de problemas, por ejemplo, el ABP, entre otros contextos prácticos y/o metodológicos). Finalmente, se evidencian esfuerzos para disponer de elementos tecnológicos como software especializado que apoyen los procesos investigativos y curriculares.

\section{Referencias}

Bain, K. (2007). Lo que hacen los mejores profesores de universidad. España: Universitat de Valencia.

(2014). Lo que hacen los mejores estudiantes de universidad. (1a. ed.). España: Universitat de Valencia.

Castellanos, O. (2007). Gestión tecnológica. De un enfoque tradicional a la inteligencia.. Bogotá: Facultad de Ingeniería-Universidad Nacional.

(2013). Competitividad. Apropiación y mecanismos para su fortalecimiento. p.189. Bogotá: Facultad de Ingeniería- Universidad Nacional.

García-Cabrera, A. (2015). "Emprender en economías emergentes: el entorno institucional y su desarrollo"..En: Innovar, Vol. 25, No.57, pp. 133-156.

Hernández, C. A. (2003). "Investigación e investigación formativa".. En: Nómadas, 195202.

Kalmanovitz, S. (2001). Las instituciones y el desarrollo económico en Colombia. (1a. Ed.). Bogotá: Grupo Editorial Norma.

Misas, G. (2004). La educación superior en Colombia: análisis y estrategias para su desarrollo. (1a. Ed.). Bogotá: Unibiblos, Universidad Nacional de Colombia.

Morales, M. (. (2014). "Apuntes teóricos sobre la relación entre conocimiento colectivo e instituciones". Colombia No. 31. p. 61-79. 
En: Revista Economía Institucional (1a. Ed.). Bogotá: Universidad Externado de Colombia No. 31, pp. 61-79.

Padilla Beltrán, J., Rincón Caballero, D. \& Buitrago Duarte, L. (2015). "La investigación formativadesdelateoría de las representaciones sociales en la Facultad de Estudios a Distancia de la Universidad Militar Nueva Granada".. En: Academia y Virtualidad, 8, (1), 21-34.

Parra, C. (2004). "Apuntes sobre la investigación formativa".. En: Educación y Educadores., 57-77.

Perkins, J. (2013). Review of Engineering Skills. Department for Business Innovation \& Skills, UK.

Restrepo, B. (2003). "Investigación formativa e investigación productiva de conocimiento en la universidad".. En: Nómadas, 203-211.

. (4 de 10 de 2014). http://www. javeriana.edu.co. Recuperado de http:// www.javeriana.edu.co/javeriana/vice_acad/ curriculos/documentos/Conceptos $\% 20 \mathrm{y} \% 20$ aplica

Safford, F. (1989). El ideal de lo práctico. El desafío de formar una elite técnica y empresarial en Colombia. (1ra Ed.). Bogotá: El Áncora Editores.

Yagodzinski, E. D. (22 de Noviembre de 2014). Academia. Recuperado de www.academia. edu: https://www.academia.edu/1504477/ Formative_Research_on_an_Instructional Design_Theory_for_Online_Learning Communities_A_Higher_Education_ Faculty_Development_Case 